\title{
Peripheral resolution using stationary and flickering gratings: the effects of age
}

\author{
Roger S. Anderson and D. Rodney McDowell \\ Vision Science Research Group, School of Biomedical Sciences, University of Ulster at Coleraine, Co., Londonderry BT52 1SA, \\ Northern Ireland
}

\begin{abstract}
Purpose. Currently, little or no psychophysical data exist on the decline in retinal ganglion cell density, with age or on the nature of any selective age-related loss of one cell type over another. The authors wished to determine the nature of any decline in ganglion cell density with age using measurements of peripheral grating resolution, which is directly related to ganglion cell sampling density.
\end{abstract}

Methods. We measured grating resolution at 4 different retinal locations in a group of 97 normal subjects ranging in age from eight to eighty-one years. Stimuli were either stationary or employed counterphase flicker at $30 \mathrm{~Hz}$ in order to selectively stimulate a greater proportion of either $\mathrm{P}$ or $\mathrm{M}$ ganglion cells.

Results. Mean resolution was significantly higher for stationary gratings than flickering gratings. Loss of peripheral resolution was approximately $5 \% /$ decade for stationary gratings and $6 \% /$ decade for flickering gratings. The ratio of resolution for flickering/non-flickering stimuli showed a small but insignificant decline with age.

Conclusions. There is a general decline in peripheral resolution with age, indicating a corresponding loss of retinal ganglioncell density. This age-related loss does not appear to be very selective in terms of cells that are sensitive to either stationary or flickering gratings. These results increase the diagnostic power of clinical tests that employ measures of peripheral resolution in subjects of different ages to detect diseases which cause loss of ganglion cells. Curr. Eye Res. 16: 1209-1214, 1997.

Key words: age; retinal ganglion cells; magnocellular pathway; peripheral resolution

Correspondence: Dr. Roger S. Anderson, Vision Science Research Group, School of Biomedical Sciences, University of Ulster at Coleraine, Co. Londonderry BT52 1SA, N. Ireland; e-mail: rs.anderson@ulst.ac.u

\section{Introduction}

Previous studies have shown that foveal visual acuity declines with increasing age (1-3 for reviews), and this loss of acuity has been attributed, at least in part, to neural as well as optical factors $(3,4)$. However, the extent of acuity loss attributable to neural factors alone is not clear (5), and it remains difficult to separate neural and optical losses of acuity in central vision.

Foveal resolution, under normal conditions, is limited by the optics of the eye, in that spatial frequencies higher than the resolution limit of the retina do not pass (6). As a consequence, it is possible that a significant loss of retinal sampling density could occur in the fovea before visual acuity is measurably affected. In peripheral vision, however, resolution for high contrast gratings is limited by the density of the underlying sampling array, in particular the density of the coarsest sequence in that array, the ganglion cells (7-13). Evidence for the sampling-limited nature of peripheral resolution comes from the observations of aliasing that can be demonstrated in peripheral vision when a subject can detect contrast in a grating stimulus but can not correctly identify target orientation or drift direction (7, 9, 14-16). From Shannon's sampling theorem (17), assuming a square array, the minimum angle of resolution (MAR) is equal to the spacing of the retinal ganglion cells (8). This means that measurements of peripheral resolution for stationary gratings may be used to obtain estimates of localised ganglion cell density. Comparison of psychophysical measurements of peripheral resolution, with predicted resolution performance based on anatomical counts of ganglion cell density, have yielded good qualitative and quantitative agreement (8, $12,13,18-20)$, indicating the value of peripheral resolution measurements in estimating localised ganglion cell density.

Previous anatomical studies have indicated a general decline in ganglion cell density with age (21-27) and also a large variation in the absolute number of ganglion cells between subjects of similar age. The anatomical study of Curcio and Allen (28) not only indicated a significant variation in the total number of ganglion cells between individuals, but also in the numbers of ganglion cells at different retinal locations in the same indi- 
vidual. Measurements of peripheral grating resolution should not only demonstrate a corresponding decrease with age, but also indicate localised retinal variations in performance.

Furthermore, different types of ganglion cells respond differently to different types of visual stimuli. Resolution for high-contrast stationary gratings is mediated by the ganglion cells that project to the parvocellular layers of the lateral geniculate nucleus ( $\mathrm{P}$ cells), which are more sensitive to stationary stimuli $(29,30)$ and are decreasingly involved in the resolution task when temporal frequency exceeds $10 \mathrm{~Hz}(12,31)$. Vision for stimuli that phase-reverse at high temporal frequency relies on the magnocellular system ( $\mathrm{M}$ cells), which is more sensitive to higher temporal frequencies $(29,32-35)$. Some debate exists as to whether or not there is a selective loss of function of one cell type over the other with age (see Spear [5] for review). A high contrast grating which counterphases at $30 \mathrm{~Hz}$ still yields sampling-limited resolution performance in peripheral vision (12) and can, therefore, be used to measure the resolution, and hence density, of a group of ganglion cells that contains a higher proportion of $\mathrm{M}$ cells.

The sampling-limited nature of peripheral resolution has important clinical implications, in that measurements of peripheral resolution could be used for the detection of eye conditions that cause death or dysfunction of retinal ganglion cells, e.g. glaucoma. Resolution measurements using gratings which phase-reverse at high temporal frequency may be particularly useful, since it has been advocated that $\mathrm{M}$ cells are damaged earliest in glaucoma (21). Anderson and O'Brien (36) measured peripheral resolution at twelve retinal locations in age-matched groups of normals, ocular hypertensives and glaucoma patients, using gratings that were either stationary or phase-reversed at $30 \mathrm{~Hz}$. They found that mean resolution was significantly lower in glaucoma patients than ocular hypertensives and in ocular hypertensives than normals, for both stationary and flickering gratings. Interestingly, they also noted that the ratio of resolution with/without flicker was also significantly lower in glaucoma patients than ocular hypertensives and in ocular hypertensives than normals, providing psychophysical evidence for a selective loss of flickersensitive cells ( $\mathrm{M}$ cells) in glaucoma. Determining the presence or absence of any selective loss with increasing age would improve the clinical utility of such a test, when measuring peripheral resolution in glaucoma-suspect patients of different ages.

For the above reasons, we wanted to measure peripheral resolution at different retinal locations in a large group of normal subjects of different ages. This would yield data on the expected normal resolution values at different retinal locations for subjects of different ages, and would determine whether or not there is any decline in retinal ganglion-cell density with increasing age. In addition, by using gratings that are either stationary or phase-reverse at $30 \mathrm{~Hz}$, we should be able to determine if there is a selective loss of $\mathrm{P}$ or $\mathrm{M}$ cells with age.

\section{Materials and methods}

\section{Apparatus}

We constructed a resolution perimeter similar to that of Anderson et al. (36), which this time measured grating resolution at four locations in the visual field, for both stationary $(0 \mathrm{~Hz})$ and phase-reversing $(30 \mathrm{~Hz})$ gratings. The stimuli were circular patches of high contrast $(90 \%)$ sinusoidal grating with the same mean luminance as the surround $\left(70 \mathrm{~cd} / \mathrm{m}^{2}\right)$, and were generated on a 17 inch high-resolution computer monitor (Eizo), using a visual stimulus generator VSG2/3 (Cambridge Research Systems). Stimuli included grating patches of 4.5 degrees diameter at 20 degrees eccentricity in each of the four major oblique meridians. The fixation point was a 1-degree red cross in the centre of the screen.

\section{Subjects}

The procedure was conducted on ninety-seven volunteer subjects ranging in age from eight to eighty-one years, drawn from the ophthalmic practice of one of the authors (DRM). All subjects underwent ocular examination prior to the experiment. All eyes included in the study had visual acuity of $6 / 6$ or better, with no observable lens opacity, intraocular pressure below 21 $\mathrm{mm} \mathrm{Hg}$, normal-appearing optic discs with vertical cup/disc ratio below 0.4 , and no visual field defect as measured by multiple stimulus supra-threshold perimetry. In addition, we excluded diabetic patients and any patient with a history of ocular disease or surgery.

\section{Psychophysical methods}

Subjects sat with their chin on a chin rest at $0.33 \mathrm{~m}$ from the screen while viewing the fixation cross. Only the left eyes of all subjects were tested. Subjects were optically corrected for the distance of the screen using full aperture reading spectacles, and the eye not in use was patched. Room lights were on and undilated pupils were employed throughout. Pupil size ranged from 3-8 $\mathrm{mm}$ between subjects, under the conditions of the experiment. Stimuli were presented randomly, in terms of location, orientation and temporal frequency, at each of the 4 locations, using a two-alternative, forced choice (2AFC) psychophysical procedure where the observer had to indicate whether the orientation of the grating was horizontal or vertical by pressing one of two buttons. Gratings oriented horizontally and vertically on the computer screen are oriented obliquely with respect to the fovea, when placed in one of the four major oblique meridians. Unlike gratings oriented radially or tangentially with respect to the fovea, such stimuli will yield equal resolution performance $(18,37)$, and so remove a potential cue to deciding orientation. Stimulus duration was $0.5 \mathrm{~s}$ and the subject had five s to respond; otherwise the test went to the next stimulus and repeated the previous stimulus at the end of the set of 4 presentations. Pressing one of the two response buttons triggered the next stimulus. All 8 stimuli were presented 24 times. Randomly interleaving the stationary presentations with the phase-reversing presentations removed any learning effects that would have resulted, if they had been run in separate sessions. Three correct responses for a particular stimulus caused a 10\% increase in spatial frequency, and one incorrect response caused a 10\% decrease in spatial frequency. This gave, on average, 4 reversals for each stimulus. Resolution threshold for each stimulus was calculated as the mean of the reversal val- 
ues. The mean resolution for all 4 locations, for both stationary and phase-reversing gratings, was then calculated for each subject.

Subjects were given a short practice period, lasting about 3 $\min$, to familiarize themselves with the procedure. Each test lasted an average of 4-5 min.

\section{Results}

Figure 1 shows the resolution values at each visual field location, averaged across all subjects, for the stationary stimuli (values to left of each stimulus) and the phase-reversing stimuli (values to right of each stimulus). The ratio of resolution with/ without $30 \mathrm{~Hz}$ phase reversal was determined at each visual field location in each subject, and the mean ratio across subjects at each visual field location is represented by the value below each stimulus.

For the stationary stimuli, resolution was higher in the inferiortemporal field (5.33 \pm 0.17 cycles/degree) than at any of the other three locations. It can also be seen that resolution was significantly higher in the temporal field than the nasal field as a whole $(5.175 \pm 0.13 \mathrm{c} / \mathrm{deg}$ [mean of two left points] cf. $4.98 \pm$ $0.12 \mathrm{c} / \mathrm{deg}$ [mean of two right points] $(\mathrm{p}<0.05$, Student's ttest]). Resolution was also significantly higher in the inferior field than the superior field as a whole $(5.22 \pm 0.13 \mathrm{c} / \mathrm{deg}$ [mean of two lower points] cf. $4.93 \pm 0.13 \mathrm{c} / \mathrm{deg}$ [mean of two upper points; $\mathrm{p}<0.05]$ ).

The average resolution values for the $30 \mathrm{~Hz}$ phase-reversing stimuli in Figure 1 (to right of each stimulus) indicate that, as with stationary gratings, resolution was higher in the inferiortemporal field ( $3.87 \pm 0.15$ cycles/degree) than at other locations. Resolution was again significantly higher in the temporal field than the nasal field as a whole $(3.63 \pm 0.12 \mathrm{c} / \mathrm{deg}$ [mean of

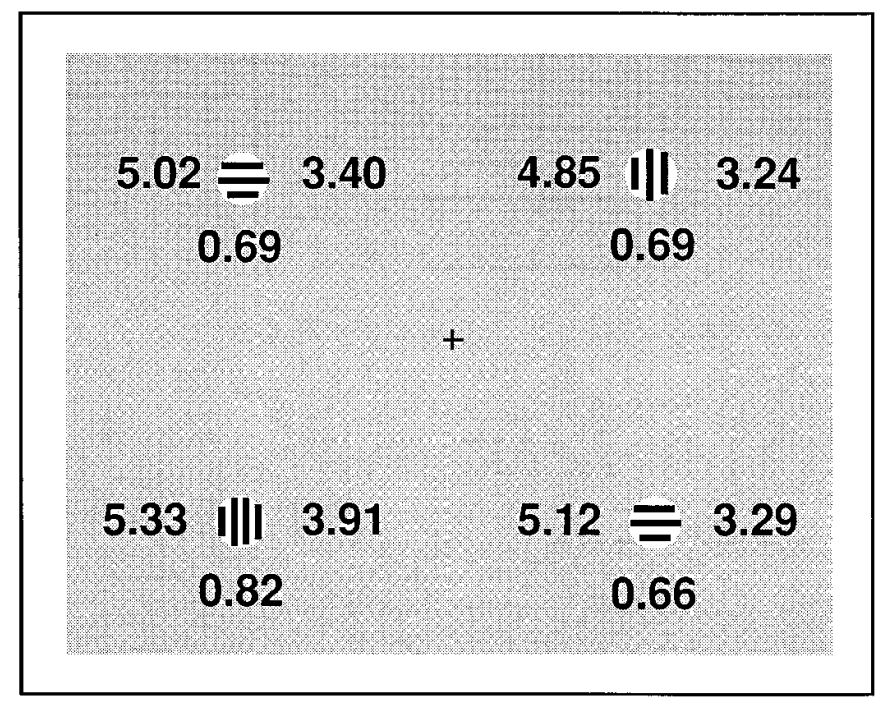

Figure 1. M ean resolution (cycles/degree) across subjects for each of the four visual field locations for: stationary gratings (to left of each stimulus), gratings phase-reversing at $30 \mathrm{~Hz}$ (to right of each stimulus), and the ratio of resolution with/without phase reversal (below each stimulus). two left points] cf. $3.26 \pm 0.10 \mathrm{c} / \mathrm{deg}$ [mean of two right points; $\mathrm{p}<0.05]$ ). Resolution was also significantly higher in the inferior field than the superior field as a whole $(3.58 \pm 0.11 \mathrm{c} / \mathrm{deg}$ [mean of two lower points] cf. $3.32 \pm 0.12 \mathrm{c} / \mathrm{deg}$ [mean of two upper points; $\mathrm{p}<0.05]$ ).

The ratio values of resolution with/without $30 \mathrm{~Hz}$ phase reversal (below each stimulus), interestingly, indicate that the ratio was again higher in the inferior-temporal field $(0.82 \pm$ 0.03 ) than at the other three locations. No significant differences in the ratios were observed between superior and inferior fields or temporal and nasal fields.

Figure $2 \mathrm{a}$ is a plot of the mean resolution across all four locations for each subject, plotted against age for the stationary stimuli. The data display a negative correlation with age $(\mathrm{r}=$ 0.53 ), which is significantly different from zero at the $5 \%$ level. Resolution falls from 5.95 cycles/degree at ten years old to 3.98 cycles/degree at eighty years old, representing a decrease of $5 \% /$ decade.

Figure $2 \mathrm{~b}$ is a plot of the mean resolution data versus age for the $30 \mathrm{~Hz}$ phase-reversing stimuli. Resolution was, on average, $40 \%$ lower for phase-reversing gratings than for stationary gratings. The data again display a negative correlation with age $(r=0.57)$, which again is significantly different from zero at the 5\% level. Resolution falls from 4.24 cycles/degree at ten years old to 2.35 cycles/degree at eighty years old, representing a fall-off of $6 \% /$ decade.

The ratio of resolution with/without $30 \mathrm{~Hz}$ phase reversal was determined at each visual field location, and the mean of the four values were calculated for each subject. This value is plotted against age for each subject in Figure 2c. The mean ratio was $0.71 \pm 0.05$ and the performance ratio showed only a weak correlation with age $(r=0.08)$.

\section{D iscussion}

The direct relationship between grating acuity and retinalganglion-cell density in the peripheral retina means any decline in ganglion-cell-sampling density with age should give rise to a corresponding decline in peripheral grating-resolution acuity. Such a decline is in evidence here. However, it could be postulated that other factors could contribute to a decline in peripheral acuity with age.

Lenticular and/or general retinal-sensitivity changes can be expected to cause an overall vertical decline in the contrastsensitivity function and hence, foveal resolution acuity. However, peripheral acuity is a different situation. Thibos et al. (11), examining peripheral acuity, found that while detection acuity declined steadily as contrast decreased, there was no change in resolution performance until contrast was reduced to 10 percent. Below this contrast level, resolution was no longer sampling-limited, but became contrast-limited, as in the fovea. Resolution acuity is apparently unaffected by changes in contrast, as long as contrast remains above a certain minimum for sampling limited performance. Subjects in this study were chosen to have minimal lens opacity and good central acuity, so that grating stimuli would remain clearly above detection threshold throughout the test, even for the older subjects. 

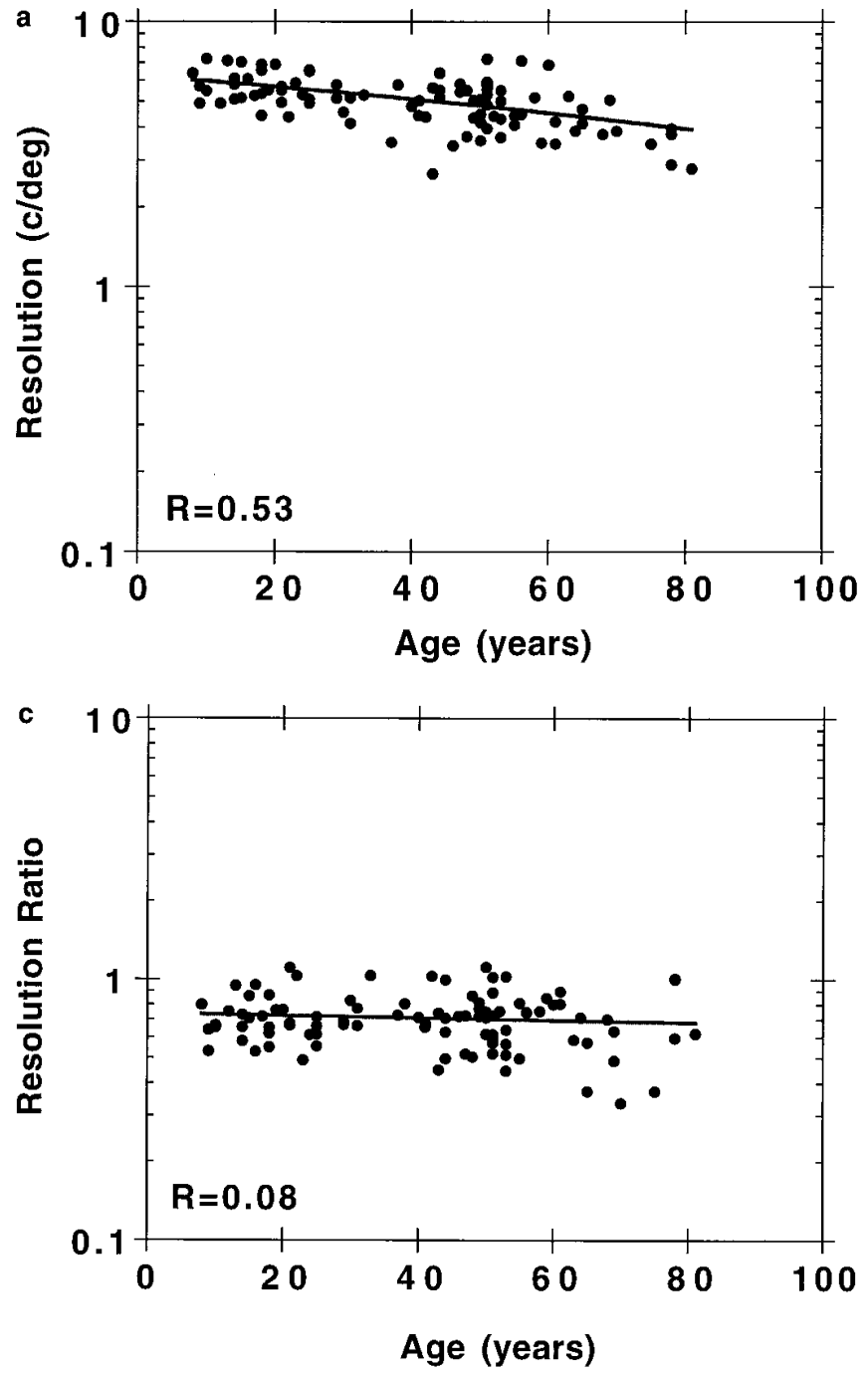

Therefore, it is unlikely that resolution performance was affected by age-related lenticular changes or variations in pupil size or retinal-contrast sensitivity.

It should also be noted that we used undilated pupils throughout this study. Pupil size varies with age (38), leading to decreased retinal illuminance and possibly reduced acuity. However, previous studies of foveal-contrast sensitivity have shown no significant reduction in performance with decreasing pupil size $(39,40)$, especially over the range of retinal illuminances, in this study. In addition, Elliott et al. (41) found that induced pupil miosis, even in combination with increased light absorption, caused no significant decline in foveal-contrast sensitivity. We would expect pupil-size variations and minor lenticular changes to have an even smaller effect in the periphery.

The foregoing measurements of resolution also show good qualitative agreement with the anatomical study of Curcio and Allen (28), which indicated that ganglion cell density was different in different meridians. Density was generally higher in the superior retina (inferior field) than the inferior retina (superior field) and in the nasal retina (temporal field) than the temporal retina (nasal field). This should mean that resolution is correspondingly better in the inferior field than the superior

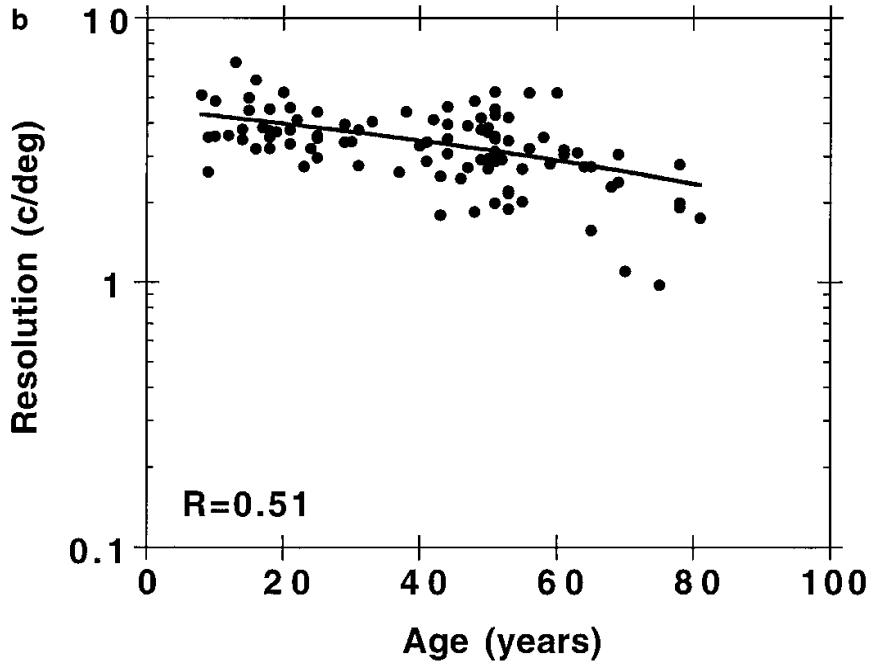

Figure 2. M ean resolution for each subject plotted against age. (a) Stationary gratings, (b) G ratings phase-reversing at $30 \mathrm{~Hz}$, and (c) $\mathrm{R}$ atio of resolution with/without phase reversal.

field and in the temporal field than the nasal field. The resolution values for stationary stimuli in Figure 1 confirm this prediction, with resolution performance being highest in the inferiortemporal field. Assuming that resolution performance for high contrast, stationary stimuli in Figure 1 is mediated by the parvocellular ganglion cells that represent about $80 \%$ of the total number of retinal ganglion cells $(42,43)$, we converted the anatomical counts of ganglion cell density of Curcio and Allen (28) to a first approximation, psychophysical resolution performance. This gave us a predicted mean resolution performance at 20 degrees eccentricity of 5.5 cycles/degree, which is very close to the mean psychophysical performance of 5.1 cycles/ degree. (We assumed a square array as Thibos (8), MAR $=1$ / density; a noisy hexagonal array might be a more realistic assumption and may yield slightly higher resolution predictions (44)).

The rate of loss of cells here is also in agreement with previous histological measures of age-related ganglion-cell loss, which report cell loss at a rate of between 3 and $6 \% /$ decade $(22,24,25)$.

The resolution values in Figure 1 for the $30 \mathrm{~Hz}$ phase-reversing stimuli represent performance, mediated by a population of 
ganglion cells comprising a higher number of $\mathrm{M}$ ganglion cells, which are more sensitive to high temporal frequencies than P ganglion cells (29). Resolution in this case is significantly lower at all locations than for the stationary stimuli: $26 \%$ lower on average. However, performance again is higher in the inferior field than the superior field and in the temporal field than the nasal field, indicating that the distribution of cells sensitive to high temporal frequency gratings is qualitatively similar to that of cells sensitive to stationary gratings.

The decline in peripheral resolution with age, for stationary stimuli (Fig. 2a), indicates a general decline in responding ganglion-cell sampling density, in particular, the parvocellular ganglion cells, which are sensitive to this type of stimulus. The decline in peripheral resolution with age for $30 \mathrm{~Hz}$ phasereversing gratings (Fig. 2b) indicates that the ganglion cell population which responds to this high temporal frequency stimulus, comprising a high proportion of $\mathrm{M}$ cells, also declines with age. This information is of direct clinical relevance, in that it helps to establish age-related normal values for any test that seeks to measure ganglion cell loss in diseased eyes although, as with previous anatomical studies, there appears to be a large inter-individual variation in cell number, even in subjects of similar age.

The study of Anderson and O'Brien (36) found that the ratio of resolution with/without $30 \mathrm{~Hz}$ flicker was significantly lower in glaucoma patients than ocular hypertensives and in ocular hypertensives than normals, indicating a selective loss of cells sensitive to the flickering stimulus. The resolution ratios for flickering/stationary gratings in the present study indicate no significant loss of one cell population over the other, due to age alone. This is in agreement with previous anatomical studies, which found no selective loss of differently sized optic nerve fibres with age $(23,24)$. This result is clinically useful, in that it indicates that any selective loss of flickersensitive cells in glaucoma patients, as found by Anderson and O'Brien, is likely to be pathological rather than age-related.

This knowledge will increase the diagnostic power of clinical psychophysical tests, which use measures of peripheral resolution to detect diseases of the eye that cause selective death or dysfunction of retinal ganglion cells.

\section{Acknowledgments}

This research was supported by an equipment grant from the Royal College of Surgeons of Edinburgh and by an Ophthalmic Research Grant from the Guide Dogs for the Blind Association (UK).

\section{R eferences}

1. Kline, D. W. and Schreiber, F. (1985) Vision and aging. In Handbook of the Psychology of Aging (Eds. Birren, J. E. and Schaie, K. W.). Pp. 296-331. Van Nostrand, New York.

2. Pitts, D. G. (1982) The effects of aging on selected visual functions: dark adaptation, visual acuity, stereopsis and brightness contrast. In Aging and Human Visual Function
(Eds. Sekuler, R., Kline, D. and Dismukes, K.). Pp. 131159. Liss, New York.

3. Weale, R. A. (1975) Senile changes in visual acuity. Trans. Ophthalmol. Soc. UK, 95, 36-38.

4. Weale, R. A. (1982) Senile ocular changes, cell death and vision. In Aging and Human Visual Function (Eds. Sekuler, R., Kline, D. and Dismukes, K.). Pp. 161-171. Liss, New York.

5. Spear, P. D. (1993) Neural bases of visual deficits during aging. Vision Res. 33, 2589-2609.

6. Campbell, F. W. and Gubisch, R. W. (1966) Optical quality of the human eye. J. Physiol. 186, 558-578.

7. Smith, R. A. and Cass, R. A. (1987) Aliasing in the parafovea with incoherent light. J. Opt. Soc. Am. A 4, 15301534.

8. Thibos, L. N., Cheney, F. E. and Walsh, D. J. (1987) Retinal limits to the detection and resolution of gratings. $J$. Opt. Soc. Am. A 4, 1524-1529.

9. Thibos, L. N., Walsh, D. J. and Cheney, F. E. (1987) Vision beyond the resolution limit: aliasing in the periphery. Vision Res. 27, 2193-2197.

10. Williams, D. R. and Coletta, N. J. (1987) Cone spacing and the visual resolution limit. J. Opt. Soc. Am. A 4, 15141523.

11. Thibos, L. N., Still, D. L. and Bradley, A. (1996) Characterization of spatial aliasing and contrast sensitivity in peripheral vision. Vision Res. 36, 249-258.

12. Anderson, R. S. (1996) Aliasing in peripheral vision for flickering gratings. J. Opt. Soc. Am. A 13, 2288-2293.

13. Anderson, R. S. (1996) The selective effect of optical defocus on detection and resolution acuity in peripheral vision. Curr. Eye. Res. 15, 351-353.

14. Williams, D. R. (1985) Aliasing in human foveal vision. Vision Res. 25, 195-205.

15. Williams, D. R. (1985) Visibility of interference fringes near the resolution limit. J. Opt. Soc. Am. A 2, 1087-1093.

16. Anderson, S. J. and Hess R. F. (1990) Post-receptoral undersampling in normal human peripheral vision. Vision Res. 30, 1507-1515.

17. Shannon, C. E. (1949) Communication in the presence of noise. Proc. IRE, 37, 10-21.

18. Anderson, R. S., Wilkinson, M. O. and Thibos L. N. (1992) Psychophysical localization of the human visual streak. Optom. Vis. Sci. 69, 171-174.

19. Dacey, D. M. (1993) The mosaic of midget ganglion cells in the human retina. J. Neurosci. 13, 5334-5355.

20. Dacey, D. M. (1994) Physiology, morphology and spatial densities of identified ganglion cell types in primate retina. In Higher-Order Processing in the Visual System, Ciba Foundation Symposium 184 (Eds. Goode, J. A. and Brocks, G. R.). Pp. 12-34. Wiley, Chichester and London.

21. Quigley, H. A., Sanchez, R. M., Dunkelberger, G. R., L'Hernault, N. L. and Baginski, T. A. (1987) Chronic glaucoma selectively damages large optic nerve fibers. Invest. Ophthalmol. Vis. Sci. 28, 913-920.

22. Balazsi, A. G., Rottman, J., Drance, S. M., Schulzer, M. and Douglas, G. R. (1984) The effect of age on the nerve 
fiber population of the human optic nerve. Am. J. Ophthalmol. 97, 760-766.

23. Dolman, C. L., McCormick, A. Q. and Drance, S. M. (1980) Aging of the optic nerve. Arch. Ophthalmol. 98, 2053-2058.

24. Johnson, B. M., Miao, M. and Sadun, A. A. (1987) Agerelated decline of human optic nerve axon populations. Age, 10, 5.

25. Jonas, J. B., Schmidt, A. M., Muller-Bergh, J. A., Schlotzer-Schrehardt, U. M. and Naumann, G. O. H. (1992) Human optic nerve fiber count and optic disc size. Invest. Ophthalmol. Vis. Sci. 33, 2012-2018.

26. Mikelberg, F. S., Drance, S. M., Schulzer, M., Yidegiligne, H. M. and Weis, M. M. (1989) The normal human optic nerve. Ophthalmol. 86, 1325.

27. Repka, M. X. and Quigley, H. A. (1989) The effect of age on normal human optic nerve fiber number and diameter. Ophthalmol. 96, 26-31.

28. Curcio, C. A. and Allen, K. A. (1990) Topography of ganglion cells in human retina. J. Comp. Neurol. 300, 5-25.

29. Derrington, A. M. and Lennie, P. (1984) Spatial and temporal contrast sensitivities of neurons in the lateral geniculate nucleus of macaque. J. Physiol. 357, 219-240.

30. Merigan, W. H. and Maunsell, J. H. R. (1993) How parallel are the primate visual pathways? Ann. Rev. Neurosci. 16, 369-402.

31. Anderson, R. S., Detkova, P. and O'Brien, C. (1995) Effect of temporal frequency and contrast on peripheral grating resolution. Curr. Eye. Res. 14, 1031-1033.

32. Hicks, T. P., Lee, B. B. and Vidyasagar, T. R. (1983) The responses of cells in the macaque lateral geniculate nucleus to sinusoidal gratings. J. Physiol. 337, 183-200.

33. Merigan, W. H. (1991) $\mathrm{P}$ and $\mathrm{M}$ specialization in the macaque. In From Pigments to Perception (Eds. Valberg, A. and Lee, B. B.). Pp. 117-125. Plenum Press, New York.

34. Merigan, W. H. and Eskin, T. A. (1986) Spatio-temporal vision of macaques with severe loss of $\mathrm{Pb}$ retinal ganglion cells. Vision Res. 26, 1751-1761.

35. Merigan, W. H. and Maunsell, J. H. (1990) Macaque vision after magnocellular lateral geniculate lesions. Vis. Neurosci. 5, 347-352.

36. Anderson, R. S. and O'Brien, C. (1996) Psychophysical evidence for a selective loss of $\mathrm{M}$ ganglion cells in glaucoma. Vision Res. 37, 1079-1083.

37. Rovamo, J., Virsu, V., Laurinen, P. and Hyvarinen, L. (1982) Resolution of gratings oriented along and across meridians in peripheral vision. Invest. Ophthalmol. Vis. Sci. 23, 666-670.

38. Winn, B., Whitaker, D., Elliott, D. B. and Phillips, N. J. (1994) Factors affecting light-adapted pupil size in normal human subjects. Invest. Ophthalmol. Vis. Sci. 35, 1132-1137.

39. Woodhouse, J. M. (1975) The effect of pupil size on grating detection at various contrast levels. Vision Res. 15, 645-648.

40. Sloane, M. E., Owsley, C. and Alvarez, S. L. (1988) Aging, senile miosis and spatial contrast sensitivity at low luminance. Vision Res. 28, 1235-1246.
41. Elliott, D. B., Whitaker, D. and MacVeigh, D. (1990) Neural contribution to spatiotemporal contrast sensitivity decline in healthy aging eyes. Vision Res. 30, 541-547.

42. Perry, V. H., Oehler, R. and Cowey, A. (1984) Retinal ganglion cells that project to the dorsal lateral geniculate in the macaque monkey. Neuroscience, 12, 1101-1123.

43. Rodieck, R. W. (1991) Which cells code for color? In From Pigments to Perception: Advances in Understanding Visual processes (Eds. Valberg, A. and Lee, B. B.). Pp. 83104. Plenum Press, New York and London.

44. Yellot, J. I. (1982) Spectral analysis of spatial sampling by photoreceptors: topological disorder prevents aliasing. Vision Res. 22, 1205-1210. 